\title{
IgG4-related Periarteritis Successfully Diagnosed by an Alternative Prostate Biopsy
}

\author{
Ryo Koda ${ }^{1}$, Masafumi Tsuchida ${ }^{1}$, Noriaki Iino ${ }^{1}$, Masaki Murata ${ }^{2}$, Kohei Inui ${ }^{2}$, \\ Yuki Nakagawa ${ }^{2}$, Tsutomu Nishiyama ${ }^{2}$, Yohei Ikeda ${ }^{3}$, Go Hasegawa ${ }^{4}$, \\ Fumiaki Oguma ${ }^{5}$ and Ichiei Narita ${ }^{6}$
}

\begin{abstract}
:
A 56-year-old man was referred to our facility after developing edema in his right leg. Enhanced computed tomography (CT) revealed a periarterial soft tissue mass surrounding the right iliac artery compressing the iliac vein. His elevated serum IgG4 level indicated IgG4-related disease (IgG4-RD). Both a percutaneous and surgical biopsy of this periarterial lesion proved challenging and were not pursued. A prostate biopsy, however, finally provided a histological diagnosis of IgG4-RD. Oral steroid therapy successfully resolved his leg edema and periarterial lesion. This case illustrates the usefulness of an alternative prostate biopsy for the histological diagnosis of IgG4-RD when approaching the primary affected lesion is difficult.
\end{abstract}

Key words: IgG4-related disease, periarteritis, prostate biopsy, prostatitis

(Intern Med 58: 2401-2406, 2019)

(DOI: 10.2169/internalmedicine.2723-19)

\section{Introduction}

IgG4-related disease (IgG4-RD) is an immune-mediated systemic fibroinflammatory condition characterized by the diffuse enlargement of affected organs and an increased serum IgG4 concentration $(1,2)$. Various clinical manifestations have been reported in IgG4-RD, as almost every organ system can be involved. Histological evidence of dense lymphoplasmacytic infiltration with IgG4+ cells $>10$ /high power field (HPF) and $\mathrm{IgG} 4+/ \mathrm{IgG}+$ cell ratio $>40 \%$, obliterative phlebitis, and distinctive fibrosis known as "storiform pattern" in affected organs is required for making a definitive diagnosis of IgG4-RD (3). However, invasive procedures for achieving a histological diagnosis are not favored if the affected lesions are difficult to approach.

We herein report a 56-year-old man who presented with newly developed unilateral edema in his right lower leg over a 1-month period. Initially, deep venous thrombosis (DVT) was suspected. However, enhanced computed tomography (CT) revealed no evidence of thrombosis. Instead, the scan showed that the right iliac vein was being compressed by an abnormal soft tissue mass around the right iliac artery. In addition, the patient had an elevated serum IgG4 level (147 $\mathrm{mg} / \mathrm{dL}$, normal range: 4.8-105), prompting suspicion of IgG4-related periarteritis. However, both a percutaneous and surgical biopsy of this periarterial lesion were considered difficult in our facility. Instead, an alternative prostate biopsy provided the histological evidence needed for a diagnosis of IgG4-RD. Subsequent steroid therapy promptly ameliorated his leg edema and reduced the periarterial soft tissue mass.

\footnotetext{
${ }^{1}$ Department of Nephrology, Uonuma Institute of Community Medicine, Niigata University Medical and Dental Hospital, Japan, ${ }^{2}$ Department of Urology, Uonuma Institute of Community Medicine, Niigata University Medical and Dental Hospital, Japan, ${ }^{3}$ Department of Radiology, Uonuma Institute of Community Medicine, Niigata University Medical and Dental Hospital, Japan, ${ }^{4}$ Department of Pathology, Uonuma Institute of Community Medicine, Niigata University Medical and Dental Hospital, Japan, ${ }^{5}$ Department of Cardiovascular Surgery, Uonuma Institute of Community Medicine, Niigata University Medical and Dental Hospital, Japan and ${ }^{6}$ Division of Clinical Nephrology and Rheumatology, Niigata University Graduate School of Medical and Dental Science, Japan

Received: January 22, 2019; Accepted: February 11, 2019; Advance Publication by J-STAGE: April 17, 2019

Correspondence to Dr. Ryo Koda, ryokouda@gmail.com
} 


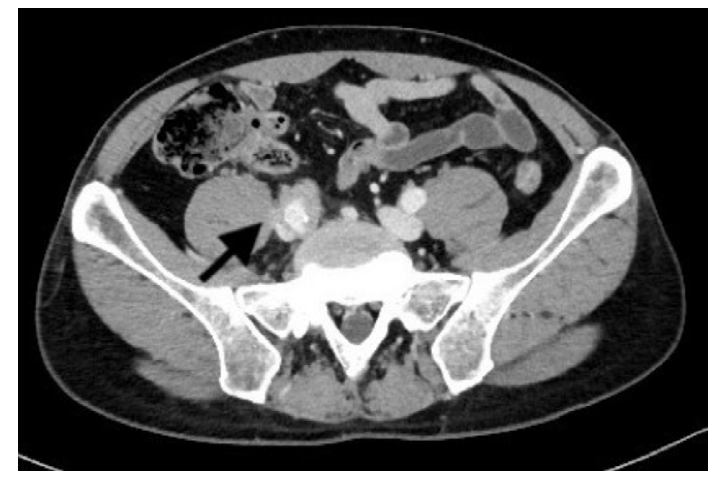

Figure 1. Enhanced CT performed at the time of referral to our Department of Cardiovascular Surgery. The right iliac vein was compressed by an abnormal periarterial soft tissue mass surrounding the right iliac artery (arrow).

\section{Case Report}

A 56-year-old man was referred to our facility due to newly developed edema in the right leg over a 1-month period. His medical history included lumbar disc herniation and laparoscopic cholecystectomy. He was not taking any regular medications or supplements. He did not regularly drink alcohol but had smoked 20 cigarettes per day from 20 to 48 years of age. He had no known food or drug allergy. His familial history was unremarkable. DVT was suspected at a local clinic, so he was referred to our Department of Cardiovascular Surgery. Enhanced CT showed no evidence of DVT but revealed an unusual soft tissue mass surrounding the right iliac artery. The right iliac vein was compressed by this soft tissue mass, leading our radiologist to suggest that this pathological lesion might have caused his leg edema (Fig. 1). The differential diagnoses included malignant lymphoma, sarcoidosis and IgG4-RD. There were no radiological abnormalities in any other organs, and hydronephrosis was not evident at this time.

The patient was referred to our nephrology department for a further evaluation. The serum $\mathrm{IgG} 4$ concentration was increased $(147 \mathrm{mg} / \mathrm{dL})$, but soluble interleukin 2 receptor and angiotensin-converting enzyme levels were normal. The kidney function was not impaired [serum creatinine $(\mathrm{sCr}) 0.76$ $\mathrm{mg} / \mathrm{dL}$, estimated glomerular filtration rate (eGFR) $82.5 \mathrm{~mL} /$ $\left.\min / 1.73 \mathrm{~m}^{2}\right]$. The results of other laboratory tests are summarized in Table. Due to the elevated serum IgG4 concentration and the CT findings, IgG4-related periarteritis was strongly suspected. One month later, he noticed dull and persistent right lower back pain. His kidney function was found to have worsened at this time ( $\mathrm{sCr} 1.22 \mathrm{mg} / \mathrm{dL}$, eGFR $49.2 \mathrm{~mL} / \mathrm{min} / 1.73 \mathrm{~m}^{2}$ ), prompting his admission to our nephrology department.

On admission, he was $161.0 \mathrm{~cm}$ tall and weighed $61.8 \mathrm{~kg}$ (body mass index, $23.8 \mathrm{~kg} / \mathrm{m}^{2}$ ). His blood pressure was $116 /$ $81 \mathrm{mmHg}$, heart rate 66/min with regular rhythm, and body temperature was $36.5^{\circ} \mathrm{C}$. His parotid glands, submandibular glands and superficial lymph nodes were not enlarged. His heart and lungs were normal on auscultation. His abdomen was soft and flat with normal bowel sounds, but knocking pain was evident at the right lower costovertebral angle. Skin rash, cyanosis, joint tenderness and swelling were absent. Pitting edema was evident unilaterally in his right leg up to the level of the groin. Neurological findings were unremarkable. CT showed newly developed hydronephrosis in his right kidney with ureteral entrapment by the aforementioned periarterial soft tissue mass that had progressively enlarged. Gallium-67 scintigraphy showed the increased diffuse uptake in this periarterial soft tissue mass and the focal uptake at the ventral side of his prostate (Fig. 2). After consulting with urologists, transurethral ureteral stent placement was planned to alleviate hydronephrosis. Regarding the periarterial lesion, a percutaneous biopsy was considered risky, and a surgical open biopsy was regarded as highly invasive in our facility.

We previously encountered an IgG4-RD case in whom a trans-perineal prostate biopsy following a detailed evaluation with magnetic resonance imaging (MRI) was useful for obtaining histological evidence of IgG4-RD (4). On evaluating our patient's lower urinary symptoms (LUTS) in more detail, he noted that he had recently felt the slight sensation of residual urine. T2-weighted MRI showed that his prostate was normal in size, indicating benign prostate hypertrophy (BPH) was unlikely as a cause of his LUTS. However, diffusion-weighted MRI showed a high-intensity area on the ventral side of the prostate (Fig. 3), which was consistent with the hot spot noted on gallium-67 scintigraphy. He therefore underwent transurethral ureter stent placement and a prostate biopsy at the same time under general anesthesia.

After ureteral stent placement, his creatinine level improved to $0.91 \mathrm{mg} / \mathrm{dL}$ (eGFR $67.7 \mathrm{~mL} / \mathrm{min} / 1.73 \mathrm{~m}^{2}$ ). A pathological examination revealed dense lymphoplasmacytic infiltration around the prostatic glands, but no obliterative phlebitis or storiform fibrosis was observed. IgG4+ cells were noted at nearly 30 per high-power field, and the IgG4+/IgG+ cell ratio was $>40 \%$ (Fig. 4). IgG4-related periarteritis was diagnosed, and he was administered oral prednisolone therapy at a dose of $0.6 \mathrm{mg} / \mathrm{kg} /$ day. Following the steroid therapy, his leg edema promptly and completely resolved. One year later, his venous compression was ameliorated with the regression of the periarterial soft tissue mass (Fig. 5). The clinical course is summarized in Fig. 6.

\section{Discussion}

The present case underscored the usefulness of an alternative prostate biopsy for obtaining histological proof of IgG4$\mathrm{RD}$ in patients with periarterial involvement in whom biopsy procedures are challenging.

Differentiation of IgG4-RD from other similar diseases, such as sarcoidosis, multicentric Castleman disease, malignant lymphoma, granulomatosis with polyangiitis and cancer, is necessary (5), so obtaining direct histological evi- 
Table. Laboratory Data at Nephrology Referral.

\begin{tabular}{|c|c|c|c|}
\hline CBC & reference & serology & reference \\
\hline $\mathrm{WBC}(/ \mu \mathrm{L})$ & $5,000(3,300-8,600)$ & C-reactive protein (mg/dL) & $0.02(0-0.14)$ \\
\hline $\mathrm{RBC}\left(\times 10^{4} / \mu \mathrm{L}\right)$ & $448(435-555)$ & Rheumatoid factor (IU/mL) & $4(0-18)$ \\
\hline Eosinophil (\%) & 1.2 & Immunoglobulin G (mg/dL) & $1,800 *(861-1,747)$ \\
\hline Hemoglobin $(\mathrm{g} / \mathrm{dL})$ & $13.5 *(13.7-16.8)$ & Immunoglobulin A (mg/dL) & $301(93-393)$ \\
\hline \multirow[t]{2}{*}{ Platelet $\left(\times 10^{3} / \mu \mathrm{L}\right)$} & $264(158-348)$ & Immunoglobulin M (mg/dL) & $66(33-183)$ \\
\hline & & Immunoglobulin G4 (mg/dL) & $147 *(4.8-105)$ \\
\hline Urine & & $\mathrm{CH} 50(\mathrm{U} / \mathrm{mL})$ & $36(30-45)$ \\
\hline Glucose & $(-)$ & Anti-nuclear antibody & $<40$ \\
\hline Protein & $(-)$ & $\mathrm{C} 1 \mathrm{q}(\mu \mathrm{g} / \mathrm{mL})$ & $<1.5(0-3)$ \\
\hline Blood & $(-)$ & MPO-ANCA (IU/mL) & $0.6(<3.5)$ \\
\hline RBC (/HPF) & $<1$ & PR3-ANCA (IU/mL) & $<0.5(<3.5)$ \\
\hline \multirow[t]{2}{*}{ WBC (/HPF) } & $<1$ & sIL2-R (U/mL) & $243(220-530)$ \\
\hline & & BJP & $(-)$ \\
\hline Chemistry & reference & IEP & $(-)$ \\
\hline Total protein $(\mathrm{g} / \mathrm{dL})$ & $7.8(6.6-8.1)$ & $\operatorname{ACE}(\mathrm{U} / \mathrm{mL})$ & $20(7-25)$ \\
\hline Albumin $(\mathrm{g} / \mathrm{dL})$ & $4.3(4.1-5.1)$ & Lysozyme $(\mu \mathrm{g} / \mathrm{mL})$ & $6(5-10)$ \\
\hline Aspartate aminotransferase (IU/L) & $22(13-30)$ & T-PSA (ng/mL) & $0.69(0-3.7)$ \\
\hline Alanine aminotransferase (IU/L) & $17(10-42)$ & & \\
\hline Alkaline phosphatase (IU/L) & 295 (106-322) & & \\
\hline Lactate dehydrogenase (IU/L) & $232(124-222)$ & & \\
\hline$\gamma$-glutamyltransferase (IU/L) & $26(13-64)$ & & \\
\hline Total bilirubin $(\mathrm{mg} / \mathrm{dL})$ & $0.6(0.4-1.5)$ & & \\
\hline Sodium $(\mathrm{mEq} / \mathrm{L})$ & $140(138-145)$ & & \\
\hline Potassium $(\mathrm{mEq} / \mathrm{L})$ & $4.2(3.6-4.8)$ & & \\
\hline Chloride (mEq/L) & $105(101-108)$ & & \\
\hline Calcium (mg/dL) & $9.3(8.8-10.1)$ & & \\
\hline Inorganic phosphate (mg/dL) & $2.4 *(2.7-4.6)$ & & \\
\hline Blood urea nitrogen (mg/dL) & $16.9(8-18.4)$ & & \\
\hline Creatinine (mg/dL) & $0.76(0.65-1.07)$ & & \\
\hline Uric acid (mg/dL) & $7.0(3.7-7.8)$ & & \\
\hline Blood sugar (mg/dL) & 97 & & \\
\hline $\operatorname{HbA1c}(\%)$ & $6.2(4.6-6.2)$ & & \\
\hline
\end{tabular}

ACE: angiotensin-converting enzyme, BJP: Bence Jones Protein, CH50: hemolytic complement activity, HbAlc: hemoglobin A1c, HPF: high-power field, IEP: immunoelectrophoresis, MPO-ANCA: myeloperoxidase-anti-neutrophil cytoplasmic antibody, PR3-ANCA: proteinase 3-anti-neutrophil cytoplasmic antibody, PSA: prostate-specific antigen, sIL-2R: soluble interleukin-2 receptor

Abnormal values are indicated by asterisks $(*)$.

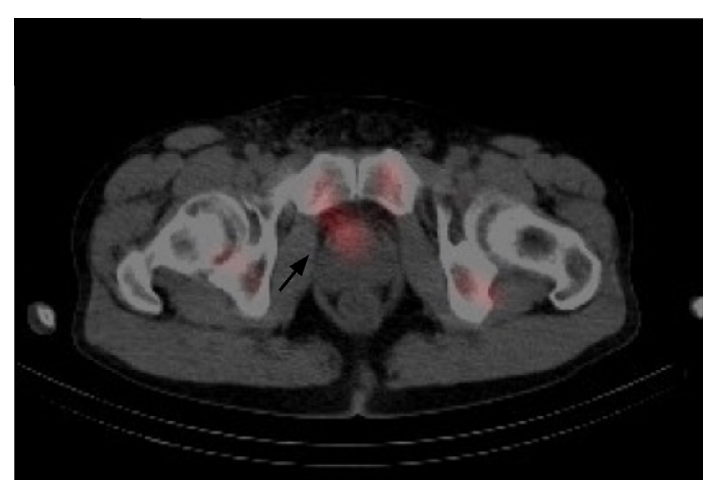

Figure 2. Gallium-67 scintigraphy. The focal uptake was noted at the ventral side of his prostate (arrow).

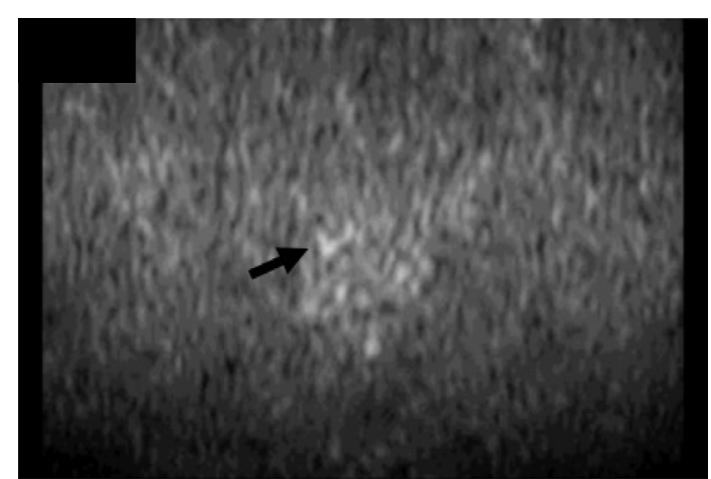

Figure 3. Diffusion-weighted MRI. Diffusion-weighted MRI showed a high-intensity area on the ventral side of prostate (arrow). 


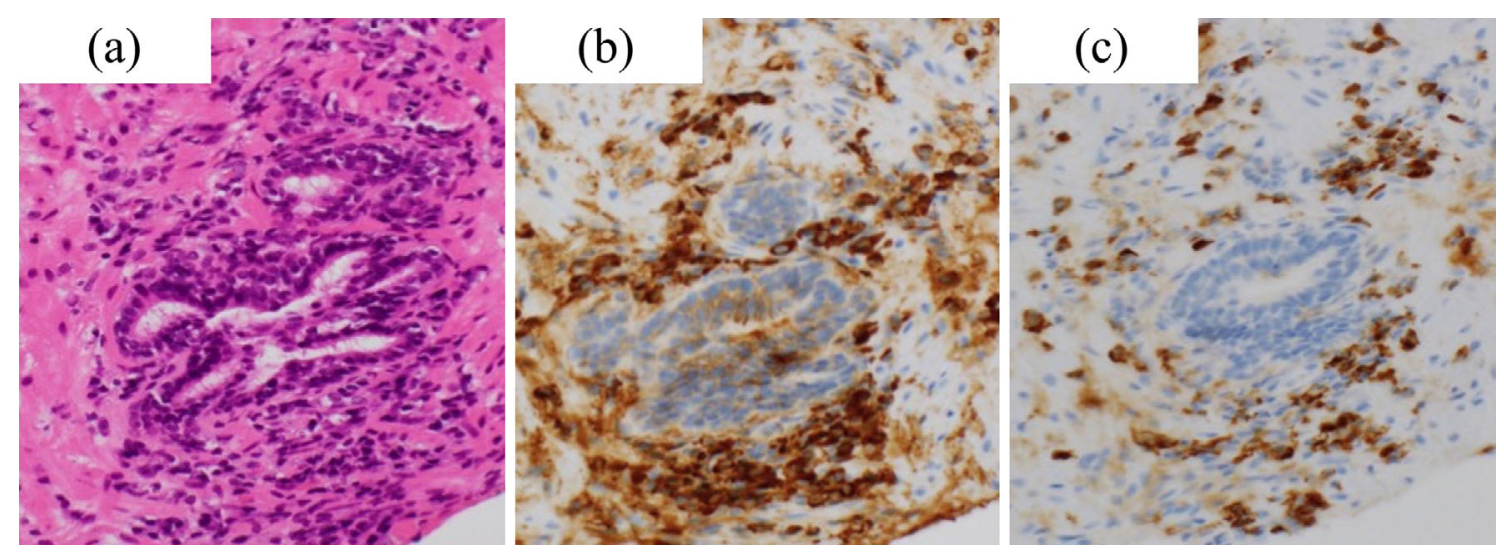

Figure 4. Prostate pathological findings. (a) Hematoxylin and Eosin staining, $\times 200$. Dense lymphoplasmacytic infiltration was observed around the prostatic glands. Obliterative phlebitis or storiform fibrosis was not observed. (b) Immunohistochemistry for IgG and (c) for IgG4. IgG4+ cells were found nearly at 30/HPF, and IgG4+/IgG+ cell ratio was $>40 \%$.

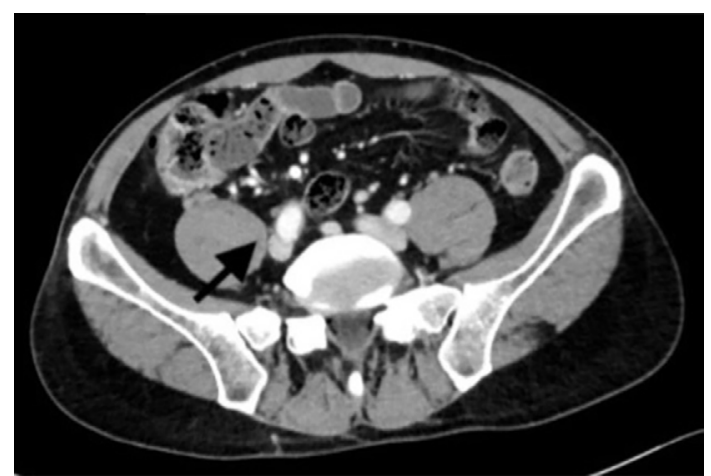

Figure 5. Enhanced CT after steroid therapy. The soft tissue mass had reduced in size, and the venous compression was ameliorated.

dence is necessary, and a biopsy of the primary affected lesion should be considered whenever possible for making a definitive diagnosis of IgG4-RD. However, a histopathological examination for some lesions is not always feasible. For example, a biopsy of a periaortic/periarterial lesion might be challenging because this procedure can be associated with fatal hemorrhagic complications. In a retrospective study that evaluated the clinical course of IgG4-related aortitis/periaortitis and periarteritis $(\mathrm{PAo} / \mathrm{PA})$, only 1 of 40 patients enrolled underwent an incisional biopsy of affected periarterial lesions for histopathological confirmation (6). That study recommended exploring other extravascular lesions where tissue sampling could be more easily and safely performed, as multiple organs can be affected simultaneously in the clinical course of IgG4-RD.

In our facility, neither a percutaneous nor surgical biopsy of the patient's periarterial lesion was favored, so other sites had to be considered. While a physical examination and CT revealed no apparent superficial organ involvement, gallium67 scintigraphy detected an increased uptake at the ventral side of our patient's prostate. We previously noted the usefulness of an alternative prostate biopsy for obtaining histo- logical proof of IgG4-RD in cases where the affected lesions are difficult to approach (4). That report describes a 79-yearold man with IgG4-related retroperitoneal fibrosis associated with acute renal failure caused by ureteral obstruction (serum IgG4: $757 \mathrm{mg} / \mathrm{dL}$ ). In addition to bilateral hydronephrosis, a high-intensity area in the prostate gland was noted on diffusion-weighted MRI, so he underwent ureteral stent placement and a prostatic needle biopsy. A histopathological examination revealed prostatitis with lymphoplasmacytic infiltration accompanied by increased numbers pf $\mathrm{IgG} 4+$ plasma cells with an $\mathrm{IgG} 4+/ \mathrm{IgG}+$ cell ratio of $25 \%$. The IgG4+/IgG+ cell ratio was not high enough in that case to meet the comprehensive criteria for IgG4-RD (>40\%) (3), but we maintain that this finding is important as evidence of IgG4-RD, since IgG4+ cells are scarcely seen in normal prostate tissue. Our findings suggest that an alternative prostate biopsy is an easy and less-invasive procedure for obtaining histological proof of IgG4-RD when the primary affected organs are difficult to approach. Our patient is the second case in whom an alternative prostate biopsy proved useful for the diagnosis of IgG4-RD.

Compared to the involvement of other organs, such as pancreas or kidney, the number of reported cases with IgG4related prostate disease is quite limited (7-14). However, the largest case control study to date suggested that prostatitis might not be such a condition, as biopsy-proven prostatitis was found in 9 out of 117 IgG4-RD patients (15). Since IgG4-related prostatitis has no specific genitourinary symptoms, it can be misdiagnosed as other common diseases, such as BPH (7). Older men are susceptible to both BPH and IgG4-RD, raising the possibility that some patients diagnosed with BPH were actually complicated with IgG4related prostatitis, and $\mathrm{BPH}$ initially considered to be a comorbid condition in IgG4-RD patients may actually have been part of the systemic involvement of IgG4-RD.

At the initial presentation, our patient did not complain of LUTS because his sensation of residual urine was slight. If gallium-67 scintigraphy had not been performed, a detailed 


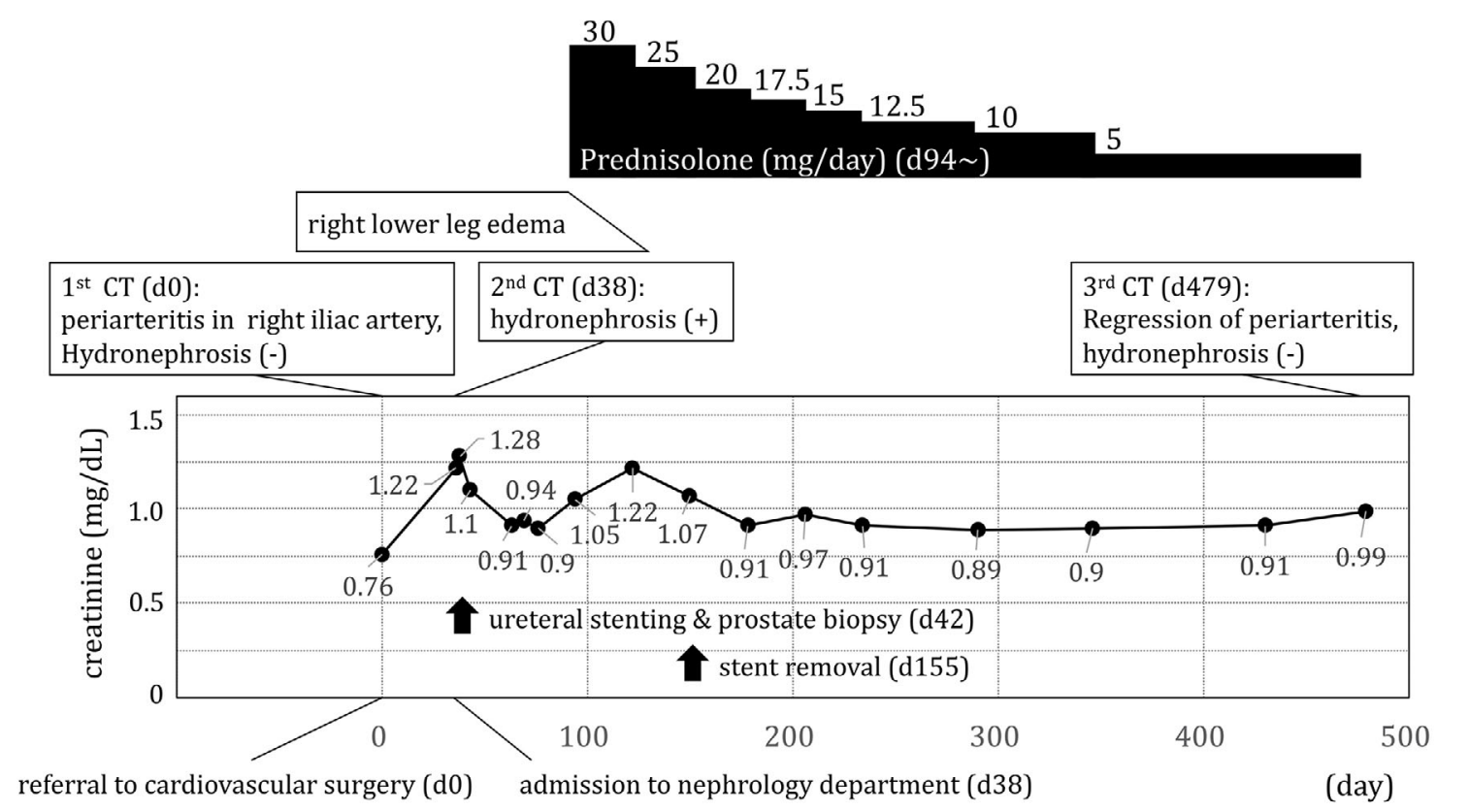

Figure 6. Clinical course. His leg edema promptly resolved with oral steroid therapy of $30 \mathrm{mg}$ daily. The ureteral stent was successfully removed, and his serum creatinine level stabilized at around 0.9 $\mathrm{mg} / \mathrm{dL}$ under $5 \mathrm{mg}$ per day of oral prednisolone.

medical history concerning his LUTS might not have been taken, and he would not have undergone a further prostatic evaluation, including MRI and a biopsy. Recently, gallium67 scintigraphy (16) and ${ }^{18} \mathrm{~F}$-fluorodeoxyglucose positron emission tomography (FDG-PET) (17) have been reported to be useful for detecting organ involvement in IgG4-RD. Notably, Takahashi et al. reported an IgG4-RD case with subclinical prostatitis (18). Their case was not accompanied by any LUTS or abnormal prostatic lesions on ultrasound sonography, but FDG-PET showed an increased uptake in his prostate, and a needle biopsy revealed lymphoplasmacytic infiltration with increased IgG4+ cells, which was compatible with IgG4-RD. As the usefulness of gallium-67 scintigraphy or FDG-PET is being increasingly recognized, the prevalence of each organ involvement in IgG4-RD will surely be clarified in the future. Considering the scarcity of reported cases, relevant specialists should be well informed about IgG4-related prostatitis, whether symptomatic or asymptomatic, when managing patients with suspected IgG 4-RD in daily clinical practice. The wider recognition of IgG4-related prostatitis and its clinical features will help confirm the safety and utility of an alternative prostate biopsy in the management of IgG4-RD.

\section{Conclusion}

An alternative prostate biopsy can provide histological evidence of IgG4-RD among patients in whom biopsies to the affected organs are challenging to perform. Relevant specialists should be well informed about IgG4-related prostatitis, whether symptomatic or asymptomatic. The safety and efficacy of an alternative prostate biopsy should be validated in larger studies to confirm its utility in the appropriate management of IgG4-RD.

The authors state that they have no Conflict of Interest (COI).

\section{References}

1. Masaki Y, Dong L, Kurose N, et al. Proposal for a new clinical entity, IgG4-positive multiorgan lymphoproliferative syndrome: analysis of 64 cases of IgG4-related disorders. Ann Rheum Dis 68: 1310-1315, 2009.

2. Umehara H, Okazaki K, Masaki Y, et al. A novel clinical entity, IgG4-related disease (IgG4RD): general concept and details. Mod Rheumatol 22: 1-14, 2012.

3. Umehara H, Okazaki K, Masaki Y, et al. Comprehensive diagnostic criteria for IgG4-related disease (IgG4-RD), 2011. Mod Rheumatol 22: 21-30, 2012.

4. Inui K, Nakagawa $\mathrm{Y}$, Watanabe $\mathrm{H}$, et al. Retroperitoneal fibrosis associated with IgG4-related disease diagnosed by prostate biopsy developed with acute post-renal renal failure: a case report. Urol Case Rep 16: 9-11, 2017.

5. Abraham M, Khosroshahi A. Diagnostic and treatment workup for IgG4-related disease. Expert Rev Clin Immunol 13: 867-875, 2017.

6. Mizushima I, Inoue D, Yamamoto M, et al. Clinical course after corticosteroid therapy in IgG4-related aortitis/periaortitis and periarteritis: a retrospective multicenter study. Arthritis Res Ther 16: R156, 2014.

7. Yoshimura Y, Takeda S, Ieki Y, Takazakura E, Koizumi H, Takagawa $\mathrm{K}$. IgG4-associated prostatitis complicating autoimmune pancreatitis. Intern Med 45: 897-901, 2006.

8. Nishimori I, Kohsaki T, Onishi S, et al. IgG4-related autoimmune prostatitis: two cases with or without autoimmune pancreatitis. Intern Med 46: 1983-1989, 2007.

9. Uehara $T$, Hamano $H$, Kawakami $M$, et al. Autoimmune pancreatitis-associated prostatitis: distinct clinicopathological entity. Pathol Int 58: 118-125, 2008. 
10. Hart PA, Smyrk TC, Chari ST. IgG4-related prostatitis: a rare cause of steroid-responsive obstructive urinary symptoms. Int $\mathrm{J}$ Urol 20: 132-134, 2013.

11. Bourlon MT, Sánchez-Ávila M, Chablé-Montero F, Arceo-Olaiz R. IgG4-related autoimmune prostatitis: is it an unusual or underdiagnosed manifestation of IgG4-related disease? Case Rep Urol 2013: 295472, 2013.

12. Nakai Y, Kagebayashi Y, Matsumoto Y, et al. IgG4-related prostatitis associated with retroperitoneal fibrosis: a case report. Hinyokika Kiyo 59: 781-784, 2013 (in Japanese, Abstract in English).

13. Li D, Kan Y, Fu F, et al. IgG4-related prostatitis progressed from localized IgG4-related lymphadenopathy. Int J Clin Exp Pathol 8: 11747-11752, 2015.

14. Jain V, Pandey PK, Bihari C. Steroid-responsive IgG4-related disease with isolated prostatic involvement: an unusual presentation with elevated serum PSA. Indian J Urol 32: 166-168, 2016.

15. Buijs J, Maillette de Buy Wenniger L, van Leenders G, et al. Im- munoglobulin G4-related prostatitis: a case-control study focusing on clinical and pathologic characteristics. Urology 83: 521-526, 2014.

16. Ishii S, Shishido F, Miyajima M, Sakuma K, Shigihara T, Kikuchi $\mathrm{K}$. Whole-body gallium-67 scintigraphic findings in IgG4-related disease. Clin Nucl Med 36: 542-545, 2011.

17. Zhao Z, Wang Y, Guan Z, Jin J, Huang F, Zhu J. Utility of FDGPET/CT in the diagnosis of IgG4-related diseases. Clin Exp Rheumatol 34: 119-125, 2016.

18. Takahashi H, Tsuboi $H$, Ogishima H, et al. [18F] fluorodeoxyglucose positron emission tomography/computed tomography can reveal subclinical prostatitis in a patient with IgG4-related disease. Rheumatology (Oxford) 54: 1113, 2015.

The Internal Medicine is an Open Access journal distributed under the Creative Commons Attribution-NonCommercial-NoDerivatives 4.0 International License. To view the details of this license, please visit (https://creativecommons.org/licenses/ by-nc-nd/4.0/).

(C) 2019 The Japanese Society of Internal Medicine Intern Med 58: 2401-2406, 2019 